\title{
The Swedish two county trial of mammographic screening for breast cancer: recent results and calculation of benefit
}

\author{
LASZLOTABAR, ${ }^{1}$ GUNNARFAGERBERG,${ }^{2}$ STEPHENW DUFFY,${ }^{3}$ ANDNICHOLAS \\ $\mathrm{EDAY}^{3}$ \\ From ${ }^{1}$ the Department of Mammography, Central Hospital, Falun, Sweden; ${ }^{2}$ Department of Radiology, University \\ Hospital, Linköping, Sweden; and ${ }^{3}$ the MRC Biostatistics Unit, Cambridge, England.
}

ABSTRACT The Swedish two county trial of breast cancer screening is now in its tenth year. This paper presents detailed results on mortality from breast cancer and from all other causes, and on the population denominators at risk for each of the first 8 years of follow up, for each county separately. These data represent a two year update on the last major report. Results show an increasingly significant deficit in deaths from breast cancer among the 77092 women invited to screening relative to the 56000 not invited $(R R=0.68, p=0.002)$, with no significant difference between the effects of screening in the two counties $(p=0.5)$. These results remain the same when adjusted for age. Analysis of all cause deaths shows no significant effect of screening $(p=0.5)$, nor was there any significant effect of screening on deaths from all causes other than breast cancer $(p=0.9)$. The rates of deaths from intercurrent illness in breast cancer cases were almost identical in the group invited to screening and the group not invited $(p=0.7)$. This result remained the same when adjusted for age. We calculate that in the age group 50-69 at entry, one breast cancer death was prevented per 4000 woman/years, per 1460 mammographic examinations, per 13.5 biopsies, and per 7.4 breast cancers detected.

The Swedish two county trial of breast cancer screening. with mammography was started in 1977 in the counties of Kopparberg and Ostergötland. The methods employed and the results of follow up until December 1984 have already been described. ${ }^{1-3}$ The 1984 results showed significantly lower breast cancer mortality among the women invited to screening. Data are now available on a further two years' follow-up, adding substantially to earlier results.

The purpose of this paper is to present in fuller form the results on mortality, stressing the relationship of screening both to breast cancer and to total mortality. These results allow one to assess both the benefit of screening and the extent of any possible adverse effects on mortality. In addition some questions about the value of breast cancer screening are addressed. ${ }^{4-9}$ These include concern about the overall mortality rate among women invited to screening (with the related question of deaths from intercurrent illness among women who developed breast cancer), the reduction in mortality from breast cancer in age subgroups and the comparison of results in the two counties.

The trial, as originally analysed, contained women who had previously had breast cancer among the population at risk (although not among the breast cancer cases or deaths as they were excluded at that stage). This imperfection has now been rectified and a definitive analysis is possible.

Finally, it is possible that the slight difference in the age distributions of the study and control groups might have modifying effects on the results, since randomisation took place at the level of small communities in this trial. Although the age distributions of the study and control groups were closely similar, an analysis which takes into account the potential age affects, in addition to the updated information, is timely.

\section{Methods}

The design and management of the two county trial have been described elsewhere. ${ }^{1}$ The numbers of individuals in the trial are shown tabulated by age and county in table 1. It is important to note that the control group is slightly younger than the group allocated to screening. Randomisation into the trial took place in the period 1977-80 and data are now available on an average follow up of 7.9 years per individual, with a minimum follow up of 5.9 years. To achieve similar total follow up times in each county, 
Table 1 Age distribution in the two counties and study groups of the trial. Data are numbers (\%) of women randomised

\begin{tabular}{|c|c|c|c|c|c|c|}
\hline \multirow[b]{3}{*}{ Age } & \multicolumn{6}{|l|}{ County } \\
\hline & \multicolumn{3}{|c|}{ Kopparberg } & \multicolumn{3}{|c|}{ Ostergötland } \\
\hline & $A S P^{I}$ & $P S P^{2}$ & Total & $A S P$ & $P S P$ & Total \\
\hline $40-49$ & $\begin{array}{l}9582 \\
(24 \cdot 8)\end{array}$ & $\begin{array}{l}5031 \\
(27 \cdot 1)\end{array}$ & $\begin{array}{l}14613 \\
(25 \cdot 6)\end{array}$ & $\begin{array}{l}10262 \\
(26 \cdot 7)\end{array}$ & $\begin{array}{l}10573 \\
(28 \cdot 3)\end{array}$ & $\begin{array}{l}20835 \\
(27 \cdot 4)\end{array}$ \\
\hline $50-59$ & $\begin{array}{l}11728 \\
(30.4)\end{array}$ & $\begin{array}{l}5557 \\
(29.9)\end{array}$ & $\begin{array}{l}17285 \\
(30 \cdot 2)\end{array}$ & $\begin{array}{l}11757 \\
(30 \cdot 5)\end{array}$ & $\begin{array}{l}11248 \\
(30 \cdot 1)\end{array}$ & $\begin{array}{l}23005 \\
(30 \cdot 3)\end{array}$ \\
\hline $60-69$ & $\begin{array}{l}11973 \\
(31 \cdot 0)\end{array}$ & $\begin{array}{l}5555 \\
(29 \cdot 9)\end{array}$ & $\begin{array}{l}17528 \\
(30-7)\end{array}$ & $\begin{array}{l}11439 \\
(29 \cdot 7)\end{array}$ & $\begin{array}{l}10714 \\
(28 \cdot 6)\end{array}$ & $\begin{array}{l}22166 \\
(29 \cdot 2)\end{array}$ \\
\hline $70-74$ & $\begin{array}{l}5306 \\
(13.8)\end{array}$ & $\begin{array}{l}2439 \\
(13 \cdot 1)\end{array}$ & $\begin{array}{l}7745 \\
(13 \cdot 5)\end{array}$ & $\begin{array}{l}5033 \\
(13 \cdot 1)\end{array}$ & $\begin{array}{l}4868 \\
(13 \cdot 0)\end{array}$ & $\begin{array}{l}9905 \\
(13 \cdot 1)\end{array}$ \\
\hline Total & 38589 & 18582 & 57171 & 38491 & 37403 & 75921 \\
\hline
\end{tabular}

${ }_{1}^{1} \mathrm{ASP}=$ Active study population, ie, invited to screening

2 PSP $=$ Passive study population, ie, not invited to screening

data are presented on follow up to December 1986 in Kopparberg and December 1987 in Östergötland. A death was classified as being from breast cancer if breast cancer was considered by the project group to be a primary or contributing cause of death. The detailed guidelines used in assessing cause of death are given as an appendix. Statistical analysis of mortality was based on Poisson log-linear modelling, using person-years at risk as an offset. ${ }^{10}$ Deviance $\chi^{2}$ statistics were used to assess the significance of differences, incorporating adjustments for county and age at randomisation as necessary. Relative risks and confidence intervals on these are presented.

For brevity, women invited to screening are referred to in the text as the active study population (ASP) and women not invited (the control group) as the passive study population (PSP).

\section{Results}

BREAST CANCER MORTALITY

Numbers of breast cancer deaths and the results of crude analysis, ignoring age are shown in table 2 , for follow up to the end of 1984, 1985 and 1986. The relative risks have remained roughly the same, as the significance has increased with increasing numbers of deaths, and the confidence intervals on the relative risks have narrowed. Note that the difference achieves statistical significance in each county separately in $\rightleftharpoons$ 1986/87. Although the relative risks for Östergötland county were slightly higher than for Kopparberg, the differences were not significant (see last column of table 2 and note the confidence intervals). In Ostergötland, stage at presentation is slightly earlier in the control group (38\% stage 1 rather than $34 \%$ ) and

Table 2 Breast cancer deaths and results of crude analysis for follow up data to end of 1984, 1985 and 1986

\begin{tabular}{|c|c|c|c|c|c|c|c|c|c|c|c|c|c|}
\hline \multirow[b]{3}{*}{$\begin{array}{l}\text { Follow } \\
\text { up to }\end{array}$} & \multirow[b]{3}{*}{$\begin{array}{l}\text { Study } \\
\text { group }\end{array}$} & \multicolumn{12}{|l|}{ County } \\
\hline & & \multicolumn{4}{|c|}{ Kopparberg } & \multicolumn{4}{|c|}{ Ostergötland } & \multirow[b]{2}{*}{$\begin{array}{l}\text { Combined } R R^{3} \\
(95 \% C I)\end{array}$} & \multirow[b]{2}{*}{$\chi^{2}$} & \multirow[b]{2}{*}{$\operatorname{Sign}^{3}$} & \multirow[b]{2}{*}{$\begin{array}{l}\text { Inter- } \\
\text { action }\end{array}$} \\
\hline & & Deaths & Population & $\begin{array}{l}R R^{I} \\
(95 \% C I)\end{array}$ & $\operatorname{Sign}^{2}$ & Deaths & Population & $\begin{array}{l}\operatorname{RR}^{I} \\
(95 \% C I)\end{array}$ & $S i g n^{2}$ & & & & \\
\hline 1984 & $\begin{array}{l}\text { ASP } \\
\text { PSP }\end{array}$ & $\begin{array}{l}51 \\
39\end{array}$ & $\begin{array}{l}38589 \\
18582\end{array}$ & $\begin{array}{c}0.63 \\
(0.41,0.96)\end{array}$ & 0.03 & $\begin{array}{l}36 \\
47\end{array}$ & $\begin{array}{l}38491 \\
37403\end{array}$ & $\begin{array}{c}0.74 \\
(0.48,1 \cdot 15)\end{array}$ & 0.2 & $\begin{array}{c}0 \cdot 68 \\
(0 \cdot 50,0 \cdot 93)\end{array}$ & $6 \cdot 1$ & 0.01 & 0.6 \\
\hline 1985 & $\begin{array}{l}\text { ASP } \\
\text { PSP }\end{array}$ & $\begin{array}{l}71 \\
52\end{array}$ & $\begin{array}{l}38589 \\
18582\end{array}$ & $\begin{array}{c}0.66 \\
(0.45,0.94)\end{array}$ & 0.02 & $\begin{array}{l}53 \\
67\end{array}$ & $\begin{array}{l}38491 \\
37403\end{array}$ & $\begin{array}{c}0.77 \\
(0 \cdot 53,1 \cdot 11)\end{array}$ & $0 \cdot 1$ & $\begin{array}{c}0 \cdot 71 \\
(0 \cdot 54,0 \cdot 92)\end{array}$ & $6 \cdot 8$ & 0.009 & 0.5 \\
\hline $1986^{5}$ & $\begin{array}{l}\text { ASP } \\
\text { PSP }\end{array}$ & $\begin{array}{l}77 \\
58\end{array}$ & $\begin{array}{l}38589 \\
18582\end{array}$ & $\begin{array}{c}0.64 \\
(0.45,0.90)\end{array}$ & 0.008 & $\begin{array}{l}83 \\
109\end{array}$ & $\begin{array}{l}38491 \\
37403\end{array}$ & $\begin{array}{c}0.74 \\
(0.55,0.99)\end{array}$ & 0.04 & $\begin{array}{c}0.70 \\
(0 \cdot 55,0 \cdot 87)\end{array}$ & 10.4 & 0.001 & 0.5 \\
\hline
\end{tabular}

1 Relative risk of death from breast cancer for active study population (ASP) $v$ passive study population (PSP)

$2 \mathrm{p}$ value for $\chi^{2}$ test for difference in risk between ASP and PSP

3 Adjusted for county

$4 \mathrm{p}$ value for $\chi^{2}$ test for a difference in the effect of screening between the counties

5 For Ostergötland, December 1987 
the overall breast cancer mortality slightly lower, so some slight differences in the effect of screening could be expected. Had the difference between the relative risks in the two counties been close to significance, separate analyses of the two counties' data would be indicated. Since it is far from significant, the study has been analysed as it was designed, as a whole.

For a more comprehensive assessment, the mortality figures were analysed taking account of age. The breast cancer deaths in each county and study group are shown by age group and year since randomisation in table 3. Mid-year estimates of person-years at risk were calculated to form the denominators of these mortality figures. These are shown in table 4. Results of statistical analysis are shown in table 5. The deficit in breast cancer deaths associated with screening increases slightly after adjustment for the concomitant factors. Statistical significance increases considerably after adjustment, reflecting the slight difference in age structure between the two groups.

When data were analysed in individual age groups (see table 6), the effect of screening was significant in age groups $50-59$ and $60-69$, but not in age groups 40-49 and 70-74, where the number of breast cancer deaths was smaller. The effect was, however, in the same direction-a lower risk in the ASP. A formal test of heterogeneity among the groups gave a nonsignificant result. As in the county by county considerations, the result of primary importance derives from the analysis of the trial as a whole, as designed, incorporating adjustments for concomitant factors rather than splitting data up by these factors.

MORTALITY FROM CAUSES OTHER THAN BREAST CANCER Deaths from all causes except breast cancer are shown tabulated by age, county, study group and time period in table 7. These were analysed offset by the same

Table 3 Breast cancer deaths tabulated by county, study group, age and time since randomisation

\section{County}

Kopparberg

Time since randomisation
Ostergötland

Time since randomisation

Death $\begin{array}{llllllllll}0-1 & 1-2 & 2-3 & 3-4 & 4-5 & 5-6 & 6-7 & 7-8 & 9-10 & \text { Total rate }\end{array}$

Age Study 0-1 $1-2 \quad 2-3 \quad 3-4 \quad 4-5 \quad 5-6 \quad 6-7 \quad 7-8 \quad 9-10$

\begin{tabular}{|c|c|c|c|c|c|c|c|c|c|c|c|c|c|c|c|c|c|c|c|c|c|c|c|}
\hline $40-49$ & $\begin{array}{l}\text { ASP } \\
\text { PSP }\end{array}$ & $\begin{array}{l}0 \\
0\end{array}$ & $\begin{array}{l}\mathbf{0} \\
\mathbf{0}\end{array}$ & $\begin{array}{l}3 \\
0\end{array}$ & $\begin{array}{l}1 \\
1\end{array}$ & $\begin{array}{l}2 \\
1\end{array}$ & $\begin{array}{l}1 \\
0\end{array}$ & $\begin{array}{l}5 \\
3\end{array}$ & $\begin{array}{l}0 \\
3\end{array}$ & $\begin{array}{l}1 \\
1\end{array}$ & $\begin{array}{r}13 \\
9\end{array}$ & $\begin{array}{l}1.5 \\
2.3\end{array}$ & $\begin{array}{l}0 \\
0\end{array}$ & $\begin{array}{l}2 \\
1\end{array}$ & $\begin{array}{l}0 \\
2\end{array}$ & $\begin{array}{l}2 \\
0\end{array}$ & $\begin{array}{l}1 \\
2\end{array}$ & $\begin{array}{l}3 \\
4\end{array}$ & $\begin{array}{l}4 \\
4\end{array}$ & $\begin{array}{l}3 \\
1\end{array}$ & $\begin{array}{l}0 \\
1\end{array}$ & $\begin{array}{l}15 \\
15\end{array}$ & $\begin{array}{l}1.6 \\
1.6\end{array}$ \\
\hline $50-59$ & $\begin{array}{l}\text { ASP } \\
\text { PSP }\end{array}$ & $\begin{array}{l}1 \\
0\end{array}$ & $\begin{array}{l}0 \\
2\end{array}$ & $\begin{array}{l}3 \\
2\end{array}$ & $\begin{array}{l}3 \\
2\end{array}$ & $\begin{array}{l}4 \\
5\end{array}$ & $\begin{array}{l}3 \\
3\end{array}$ & $\begin{array}{l}2 \\
2\end{array}$ & $\begin{array}{l}2 \\
4\end{array}$ & $\begin{array}{l}2 \\
0\end{array}$ & $\begin{array}{l}20 \\
20\end{array}$ & $\begin{array}{l}2 \cdot 0 \\
4 \cdot 0\end{array}$ & $\begin{array}{l}0 \\
2\end{array}$ & $\begin{array}{l}4 \\
0\end{array}$ & $\begin{array}{l}2 \\
2\end{array}$ & $\begin{array}{l}2 \\
2\end{array}$ & $\begin{array}{l}2 \\
5\end{array}$ & $\begin{array}{l}2 \\
5\end{array}$ & $\begin{array}{l}6 \\
9\end{array}$ & $\begin{array}{l}5 \\
6\end{array}$ & $\begin{array}{l}2 \\
3\end{array}$ & $\begin{array}{l}25 \\
34\end{array}$ & $\begin{array}{l}2.4 \\
3.4\end{array}$ \\
\hline $60-69$ & $\begin{array}{l}\text { ASP } \\
\text { PSP }\end{array}$ & $\begin{array}{l}2 \\
0\end{array}$ & $\begin{array}{l}0 \\
0\end{array}$ & $\begin{array}{l}4 \\
0\end{array}$ & $\begin{array}{l}3 \\
2\end{array}$ & $\begin{array}{l}3 \\
3\end{array}$ & $\begin{array}{l}3 \\
5\end{array}$ & $\begin{array}{l}4 \\
4\end{array}$ & $\begin{array}{l}5 \\
1\end{array}$ & 1 & $\begin{array}{l}25 \\
16\end{array}$ & $\begin{array}{l}2.6 \\
3.6\end{array}$ & $\begin{array}{l}0 \\
0\end{array}$ & $\begin{array}{l}2 \\
4\end{array}$ & $\begin{array}{l}6 \\
5\end{array}$ & $\begin{array}{l}4 \\
3\end{array}$ & $\begin{array}{l}1 \\
6\end{array}$ & $\begin{array}{l}6 \\
6\end{array}$ & $\begin{array}{l}3 \\
6\end{array}$ & $\begin{array}{l}2 \\
7\end{array}$ & $\begin{array}{l}3 \\
5\end{array}$ & $\begin{array}{l}27 \\
42\end{array}$ & $\begin{array}{l}2.8 \\
4.8\end{array}$ \\
\hline $70-74$ & $\begin{array}{l}\text { ASP } \\
\text { PSP }\end{array}$ & $\begin{array}{l}0 \\
0\end{array}$ & $\begin{array}{l}2 \\
3\end{array}$ & $\begin{array}{l}3 \\
2\end{array}$ & $\begin{array}{l}1 \\
0\end{array}$ & $\begin{array}{l}4 \\
4\end{array}$ & $\begin{array}{l}3 \\
2\end{array}$ & $\begin{array}{l}3 \\
1\end{array}$ & $\begin{array}{l}1 \\
0\end{array}$ & $\begin{array}{l}2 \\
1\end{array}$ & $\begin{array}{l}19 \\
13\end{array}$ & $\begin{array}{l}5 \cdot 2 \\
6.9\end{array}$ & $\begin{array}{l}0 \\
1\end{array}$ & $\begin{array}{l}0 \\
1\end{array}$ & $\begin{array}{l}1 \\
1\end{array}$ & $\begin{array}{l}2 \\
0\end{array}$ & $\begin{array}{l}5 \\
4\end{array}$ & $\begin{array}{l}1 \\
4\end{array}$ & $\begin{array}{l}4 \\
3\end{array}$ & $\begin{array}{l}1 \\
1\end{array}$ & $\begin{array}{l}2 \\
3\end{array}$ & $\begin{array}{l}16 \\
18\end{array}$ & $\begin{array}{l}4 \cdot 2 \\
5 \cdot 3\end{array}$ \\
\hline Totals & $\begin{array}{l}\text { ASP } \\
\text { PSP }\end{array}$ & $\begin{array}{l}3 \\
0\end{array}$ & $\begin{array}{l}2 \\
5\end{array}$ & $\begin{array}{r}13 \\
4\end{array}$ & $\begin{array}{l}8 \\
5\end{array}$ & $\begin{array}{l}13 \\
13\end{array}$ & $\begin{array}{l}10 \\
10\end{array}$ & $\begin{array}{l}14 \\
10\end{array}$ & $\begin{array}{l}8 \\
8\end{array}$ & $\begin{array}{l}6 \\
3\end{array}$ & $\begin{array}{l}77 \\
58\end{array}$ & & $\begin{array}{l}0 \\
3\end{array}$ & $\begin{array}{l}8 \\
6\end{array}$ & $\begin{array}{r}9 \\
10\end{array}$ & $\begin{array}{r}10 \\
5\end{array}$ & $\begin{array}{r}9 \\
17\end{array}$ & $\begin{array}{l}12 \\
19\end{array}$ & $\begin{array}{l}17 \\
22\end{array}$ & $\begin{array}{l}10 \\
15\end{array}$ & $\begin{array}{r}7 \\
12\end{array}$ & $\begin{array}{r}83 \\
109\end{array}$ & \\
\hline Totals & & 3 & 7 & 17 & 13 & 26 & 20 & 24 & 16 & 9 & 135 & & 3 & 14 & 19 & 15 & 26 & 31 & 39 & 25 & 19 & 192 & \\
\hline
\end{tabular}

1 cumulative death rate per 1000 women

ASP = active study population; $\quad \mathbf{P S P}=$ passive study population

Table 4 Mid-year estimates of population at $\cdot$ risk

\begin{tabular}{|c|c|c|c|c|c|c|c|c|c|c|c|c|c|c|c|c|c|c|c|}
\hline \multirow[b]{3}{*}{ Age } & \multirow[b]{3}{*}{ Study } & \multicolumn{18}{|l|}{ County } \\
\hline & & \multicolumn{9}{|c|}{$\begin{array}{l}\text { Kopparberg } \\
\text { Year of trial }\end{array}$} & \multicolumn{9}{|c|}{$\begin{array}{l}\text { Ostergötland } \\
\text { Year of trial }\end{array}$} \\
\hline & & $I$ & 2 & 3 & 4 & 5 & 6 & 7 & 8 & $9+10$ & $I$ & 2 & 3 & 4 & 5 & 6 & 7 & 8 & $9+10$ \\
\hline $40-49$ & $\begin{array}{l}\text { ASP } \\
\text { PSP }\end{array}$ & $\begin{array}{l}9571 \\
5027\end{array}$ & $\begin{array}{l}9493 \\
4991\end{array}$ & $\begin{array}{l}9404 \\
4954\end{array}$ & $\begin{array}{l}9327 \\
4911\end{array}$ & $\begin{array}{l}9210 \\
4848\end{array}$ & $\begin{array}{l}9108 \\
4802\end{array}$ & $\begin{array}{l}8809 \\
4767\end{array}$ & $\begin{array}{l}6864 \\
3691\end{array}$ & $\begin{array}{l}4387 \\
2255\end{array}$ & $\begin{array}{l}10252 \\
10567\end{array}$ & $\begin{array}{l}10230 \\
10553\end{array}$ & $\begin{array}{l}10209 \\
10535\end{array}$ & $\begin{array}{l}10189 \\
10513\end{array}$ & $\begin{array}{l}10167 \\
10488\end{array}$ & $\begin{array}{l}10141 \\
10459\end{array}$ & $\begin{array}{r}9771 \\
10100\end{array}$ & $\begin{array}{l}7814 \\
8508\end{array}$ & $\begin{array}{l}5615 \\
5335\end{array}$ \\
\hline $50-59$ & $\begin{array}{l}\text { ASP } \\
\text { PSP }\end{array}$ & $\begin{array}{r}11703 \\
5545\end{array}$ & $\begin{array}{r}11606 \\
5497\end{array}$ & $\begin{array}{r}11498 \\
5446\end{array}$ & $\begin{array}{r}11395 \\
5392\end{array}$ & $\begin{array}{r}11249 \\
5324\end{array}$ & $\begin{array}{r}11097 \\
5269\end{array}$ & $\begin{array}{r}10663 \\
5220\end{array}$ & $\begin{array}{l}8250 \\
3965\end{array}$ & $\begin{array}{l}5178 \\
2467\end{array}$ & $\begin{array}{l}11740 \\
11225\end{array}$ & $\begin{array}{l}11693 \\
11178\end{array}$ & $\begin{array}{l}11640 \\
11122\end{array}$ & $\begin{array}{l}11592 \\
11066\end{array}$ & $\begin{array}{l}11534 \\
11016\end{array}$ & $\begin{array}{l}11460 \\
10957\end{array}$ & $\begin{array}{l}11056 \\
10630\end{array}$ & $\begin{array}{l}9004 \\
8876\end{array}$ & $\begin{array}{l}6536 \\
6369\end{array}$ \\
\hline $60-69$ & $\begin{array}{l}\text { ASP } \\
\text { PSP }\end{array}$ & $\begin{array}{r}11905 \\
5527\end{array}$ & $\begin{array}{r}11735 \\
5450\end{array}$ & $\begin{array}{r}11547 \\
5354\end{array}$ & $\begin{array}{r}11343 \\
5243\end{array}$ & $\begin{array}{r}11092 \\
5121\end{array}$ & $\begin{array}{r}10833 \\
5004\end{array}$ & $\begin{array}{r}10220 \\
4886\end{array}$ & $\begin{array}{l}7655 \\
3551\end{array}$ & $\begin{array}{l}4534 \\
2029\end{array}$ & $\begin{array}{l}11378 \\
10660\end{array}$ & $\begin{array}{l}11258 \\
10546\end{array}$ & $\begin{array}{ll}11 & 124 \\
10424\end{array}$ & $\begin{array}{l}10971 \\
10290\end{array}$ & $\begin{array}{l}10811 \\
10139\end{array}$ & $\begin{array}{r}10646 \\
9962\end{array}$ & $\begin{array}{r}10128 \\
9472\end{array}$ & $\begin{array}{l}8046 \\
7603\end{array}$ & $\begin{array}{l}5321 \\
5498\end{array}$ \\
\hline $70-74$ & $\begin{array}{l}\text { ASP } \\
\text { PSP }\end{array}$ & $\begin{array}{l}5237 \\
2417\end{array}$ & $\begin{array}{l}5086 \\
2350\end{array}$ & $\begin{array}{l}4927 \\
2269\end{array}$ & $\begin{array}{l}4762 \\
2180\end{array}$ & $\begin{array}{l}4565 \\
2083\end{array}$ & $\begin{array}{l}4357 \\
1993\end{array}$ & $\begin{array}{l}4004 \\
1900\end{array}$ & $\begin{array}{l}2836 \\
1359\end{array}$ & $\begin{array}{r}1518 \\
775\end{array}$ & $\begin{array}{l}4978 \\
4805\end{array}$ & $\begin{array}{l}4848 \\
4671\end{array}$ & $\begin{array}{l}4704 \\
4523\end{array}$ & $\begin{array}{l}4550 \\
4370\end{array}$ & $\begin{array}{l}4382 \\
4211\end{array}$ & $\begin{array}{l}4197 \\
4036\end{array}$ & $\begin{array}{l}3880 \\
3732\end{array}$ & $\begin{array}{l}2995 \\
2902\end{array}$ & $\begin{array}{l}1827 \\
1958\end{array}$ \\
\hline
\end{tabular}

ASP = active study population; $\quad$ PSP = passive study population 
Table 5 Results of analysis of the effect of screening on breast cancer mortality adjusted for county and age

\begin{tabular}{lcl}
\hline Factors adjusted for & $R^{I}(95 \% \mathrm{CI})$ & Significance $^{2}$ \\
\hline None & 0.70 & 0.002 \\
County & $(0.56,0.88)$ & \\
& 0.70 & 0.002 \\
County and age & $(0.56,0.88)$ & \\
& $(0.69$ & 0.001 \\
& $(0.55,0.88)$ & \\
\hline
\end{tabular}

${ }^{1}$ Relative risk of death from breast cancer for active study population $v$ passive study population, and $95 \%$ confidence intervals.

$2 \mathrm{p}$ value for test of significance of difference between breast cancer mortality in ASP and PSP.

Table 6 Results of analyses of breast cancer mortality in individual age groups

\begin{tabular}{lcl}
\hline Age group & $R^{I}(95 \% C I)$ & Significance $^{l}$ \\
\hline $40-49$ & 0.92 & 0.8 \\
$50-59$ & $(0.52,1 \cdot 60)$ & \\
& 0.60 & 0.01 \\
$60-69$ & $(0.40,0 \cdot 90)$ & \\
& 0.65 & 0.03 \\
$70-74$ & $(0.44,0.95)$ & 0.77 \\
& $(0.47,1 \cdot 27)$ & 0.3 \\
\hline
\end{tabular}

$\chi_{3}^{2}$ for heterogeneity $=2 \cdot 19, \mathrm{NS}$

1 adjusted for county and time period

mid-year population estimates as were used in the analysis of breast cancer mortality. Results of the analysis are shown in table 8 . No significant difference was observed between the groups. After adjustment for age and county, the already very small difference vanished altogether. No significant interactions were observed between study group and any of age, time or county.

\section{MORTALITY FROM ALL CAUSES}

Numbers of deaths from all causes, including breast cancer, can be obtained by adding the corresponding elements of tables 4 and 7 . These were analysed as above, using the same mid-year estimates of personyears at risk. Results are shown in table 9. What appears to be a slight excess of deaths associated with screening is seen as a slight deficit when adjusted for county and age. This is attributable to the age difference between the study groups, mentioned above. There were no significance interactions between study group and any of county, age or time period.

DEATHS FROM OTHER CAUSES AMONG ASP AND PSP BREAST CANCER CASES

Numbers of deaths from other causes, and the appropriate person-years at risk from operation to death or end of follow up, are shown in table 10, for breast cancer cases in the two groups, also classified by age. Results of statistical analysis of the mortality rates are shown in Table 11. There is a considerable excess of deaths due to other causes in the breast cancer cases diagnosed in the ASP, balanced almost entirely by an excess in the person-years at risk. The latter excess arises because more cancer cases have been diagnosed in the ASP, both earlier in calendar time and earlier in stage (and so longer survival) due to the screening. There is no significant difference between the ASP and the PSP cases in the death rates from intercurrent illness (other causes), with $(p=0.7)$ or without $(p=0.7)$ an adjustment for age. Indeed the difference is quite emphatically non-significant. Thus there is no evidence of any systematic difference in classification of deaths between the ASP and the PSP. Analyses based on the number of deaths in table 10 , rather than death rates, are incorrect.

Table 7 Mortality from causes other than breast cancer, tabulated by county, study group, age and time since randomisation

\begin{tabular}{|c|c|c|c|c|c|c|c|c|c|c|c|c|c|c|c|c|c|c|c|c|c|}
\hline \multirow[b]{3}{*}{ Age } & \multirow[b]{3}{*}{$\begin{array}{l}\text { Study } \\
\text { group }\end{array}$} & \multicolumn{20}{|c|}{ County } \\
\hline & & \multicolumn{10}{|c|}{$\begin{array}{l}\text { Kopparberg } \\
\text { Time since randomisation }\end{array}$} & \multicolumn{10}{|c|}{$\begin{array}{l}\text { Ostergötland } \\
\text { Time since randomisation }\end{array}$} \\
\hline & & $0-1$ & $1-2$ & $2-3$ & $3-4$ & $4-5$ & $5-6$ & $6-7$ & $7-8$ & $8-10$ & $\begin{array}{l}\text { Death } \\
\text { rate }\end{array}$ & $0-1$ & $1-2$ & $2-3$ & $3-4$ & $4-5$ & $5-6$ & 6-7 & $7-8$ & $8-10$ & $\begin{array}{l}\text { Death } \\
\text { rate }\end{array}$ \\
\hline $40-49$ & $\begin{array}{l}\text { ASP } \\
\text { PSP }\end{array}$ & $\begin{array}{r}16 \\
4\end{array}$ & $\begin{array}{r}18 \\
5\end{array}$ & $\begin{array}{r}21 \\
6\end{array}$ & $\begin{array}{r}18 \\
8\end{array}$ & $\begin{array}{r}29 \\
8\end{array}$ & $\begin{array}{l}15 \\
11\end{array}$ & $\begin{array}{l}20 \\
14\end{array}$ & $\begin{array}{r}17 \\
6\end{array}$ & $\begin{array}{r}21 \\
3\end{array}$ & $\begin{array}{l}21 \cdot 8 \\
16 \cdot 0\end{array}$ & $\begin{array}{l}20 \\
11\end{array}$ & $\begin{array}{l}22 \\
16\end{array}$ & $\begin{array}{l}17 \\
17\end{array}$ & $\begin{array}{l}21 \\
25\end{array}$ & $\begin{array}{l}21 \\
23\end{array}$ & $\begin{array}{l}26 \\
30\end{array}$ & $\begin{array}{l}25 \\
38\end{array}$ & $\begin{array}{l}17 \\
26\end{array}$ & $\begin{array}{l}20 \\
26\end{array}$ & $\begin{array}{l}20 \cdot 6 \\
23 \cdot 0\end{array}$ \\
\hline $50-59$ & $\begin{array}{l}\text { ASP } \\
\text { PSP }\end{array}$ & $\begin{array}{l}46 \\
23\end{array}$ & $\begin{array}{l}56 \\
31\end{array}$ & $\begin{array}{l}54 \\
27\end{array}$ & $\begin{array}{l}63 \\
27\end{array}$ & $\begin{array}{l}60 \\
25\end{array}$ & $\begin{array}{l}84 \\
33\end{array}$ & $\begin{array}{l}77 \\
30\end{array}$ & $\begin{array}{l}50 \\
21\end{array}$ & $\begin{array}{l}42 \\
24\end{array}$ & $\begin{array}{l}52.0 \\
50.3\end{array}$ & $\begin{array}{l}34 \\
44\end{array}$ & $\begin{array}{l}56 \\
48\end{array}$ & $\begin{array}{l}45 \\
62\end{array}$ & $\begin{array}{l}46 \\
46\end{array}$ & $\begin{array}{l}66 \\
47\end{array}$ & $\begin{array}{l}79 \\
61\end{array}$ & $\begin{array}{l}65 \\
77\end{array}$ & $\begin{array}{l}65 \\
61\end{array}$ & $\begin{array}{l}69 \\
57\end{array}$ & $\begin{array}{l}50.6 \\
49.7\end{array}$ \\
\hline $60-69$ & $\begin{array}{l}\text { ASP } \\
\text { PSP }\end{array}$ & $\begin{array}{r}132 \\
55\end{array}$ & $\begin{array}{r}129 \\
62\end{array}$ & $\begin{array}{r}158 \\
91\end{array}$ & $\begin{array}{r}152 \\
87\end{array}$ & $\begin{array}{r}200 \\
79\end{array}$ & $\begin{array}{r}202 \\
99\end{array}$ & $\begin{array}{r}197 \\
99\end{array}$ & $\begin{array}{r}169 \\
83\end{array}$ & $\begin{array}{r}114 \\
49\end{array}$ & $\begin{array}{l}143 \cdot 3 \\
147 \cdot 4\end{array}$ & $\begin{array}{l}121 \\
107\end{array}$ & $\begin{array}{l}118 \\
117\end{array}$ & $\begin{array}{l}142 \\
118\end{array}$ & $\begin{array}{l}154 \\
142\end{array}$ & $\begin{array}{l}162 \\
153\end{array}$ & $\begin{array}{l}162 \\
189\end{array}$ & $\begin{array}{l}191 \\
165\end{array}$ & $\begin{array}{l}191 \\
179\end{array}$ & $\begin{array}{l}153 \\
148\end{array}$ & $\begin{array}{l}140.1 \\
138.9\end{array}$ \\
\hline $70-74$ & $\begin{array}{l}\text { ASP } \\
\text { PSP }\end{array}$ & $\begin{array}{r}139 \\
42\end{array}$ & $\begin{array}{r}145 \\
77\end{array}$ & $\begin{array}{r}148 \\
70\end{array}$ & $\begin{array}{r}152 \\
89\end{array}$ & $\begin{array}{r}189 \\
81\end{array}$ & $\begin{array}{r}182 \\
82\end{array}$ & $\begin{array}{r}200 \\
91\end{array}$ & $\begin{array}{r}160 \\
61\end{array}$ & $\begin{array}{r}121 \\
32\end{array}$ & $\begin{array}{l}327 \cdot 1 \\
290 \cdot 2\end{array}$ & $\begin{array}{l}114 \\
125\end{array}$ & $\begin{array}{l}142 \\
141\end{array}$ & $\begin{array}{l}144 \\
154\end{array}$ & $\begin{array}{l}161 \\
152\end{array}$ & $\begin{array}{l}168 \\
162\end{array}$ & $\begin{array}{l}197 \\
180\end{array}$ & $\begin{array}{l}186 \\
172\end{array}$ & $\begin{array}{l}149 \\
169\end{array}$ & $\begin{array}{l}137 \\
162\end{array}$ & $\begin{array}{l}320 \cdot 2 \\
333.6\end{array}$ \\
\hline
\end{tabular}

1 cumulative death rate per 1000 women

ASP = active study population; $\quad \mathrm{PSP}=$ passive study population 
Mammographic screening for breast cancer

Table 8 Results of analysis of the effect of screening on mortality from all causes except breast cancer, adjusted for county and age

\begin{tabular}{lcl}
\hline Factors adjusted for & $R^{I}(95 \% \mathrm{CI})$ & Significance $^{2}$ \\
\hline None & 1.03 & 0.2 \\
County & $(0.98,1 \cdot 07)$ & \\
County and age & 1.02 & 0.2 \\
& $(0.98,1.06)$ & \\
& 1.00 & 0.9 \\
\hline
\end{tabular}

${ }_{1}$ Relative risk of death from any cause but breast cancer for active study population $v$ passive study population

$2 \mathrm{p} \mathrm{value} \mathrm{for} \mathrm{test} \mathrm{of} \mathrm{significance} \mathrm{of} \mathrm{difference} \mathrm{between} \mathrm{mortality} \mathrm{from} \mathrm{causes} \mathrm{other}$ than breast cancer in ASP and PSP.

Table 9 Results of analysis of the effect of screening on overall mortality from all causes, adjusted for county and age

\begin{tabular}{lcl}
\hline Factors adjusted for & $R R(95 \% \mathrm{CI})$ & Significance \\
\hline None & 1.02 & 0.3 \\
County & $(0.97,1.06)$ & \\
& 1.01 & 0.6 \\
County and age & $(0.97,1.05)$ & 0.99 \\
& $(0.95,1.03)$ & 0.5 \\
\hline
\end{tabular}

QUANTIFICATION OF THE BENEFIT OF BREAST SCREENING It is of interest to examine the cost in terms of medical procedures of the deficit in observed breast cancer deaths. This cost can be expressed in terms of breast cancer deaths prevented per year per woman invited, per mammographic examination, per biopsy or per breast cancer detected at screening.
Table 11 Results of analysis of data on deaths from other causes, among breast cancer cases

\begin{tabular}{lcl}
\hline Factors adjusted for & $R^{I}(95 \% \mathrm{CI})$ & Significance $^{2}$ \\
\hline None & 1.06 & 0.7 \\
County & $(0.75,1.49)$ & \\
County and age & 1.05 & 0.8 \\
& $(0.74,1.48)$ & \\
& $(0.75,1.49)$ & 0.7
\end{tabular}

${ }_{1}$ Relative risk among breast cancer cases of dying from a cause other than breast cancer for active study population $v$ passive study population.

2 Test for a difference between ASP and PSP with respect to risk of breast cancer cases dying from another cause.

The overall deficit in breast cancer deaths 9 years after the start of the programme in each county can be derived from table 2 . One hundred and fifty nine breast cancer deaths occurred in the study group, representing a $31 \%$ reduction in the rate; 231.4 deaths would have been expected, giving a deficit of 71.4 . That is, over the 8 years, there was a deficit of eight deaths per year among approximately 77000 women allocated to screening, or one death per year per 9600 women allocated to screening. The bulk of the benefit of screening was observed in women aged 50-69, and in the latter 5 years after entry; in years 4-9 after the start of the study, among women aged 50-69 years at entry, there was approximately one breast cancer death avoided per year per $\mathbf{4 0 0 0}$ women allocated to screening ( 58 deaths prevented among 47000 women).

In women aged 50-69 years, the average interscreening interval was 33 months and compliance was $90 \%$. We assume that almost all of the benefit seen in

Table 10 Numbers of deaths from causes other than breast cancer among breast cancer cases, with person-years at risk ${ }^{1}$, by study group and age in Kopparberg county

\begin{tabular}{|c|c|c|c|c|c|c|}
\hline \multirow[b]{2}{*}{ Age group } & \multicolumn{3}{|l|}{$A S P$} & \multicolumn{3}{|l|}{ PSP } \\
\hline & Deaths & Person-years & Rate/100p.year & Deaths & Person-years & Rate/100p.year \\
\hline \multicolumn{7}{|c|}{ (a) Kopparberg county } \\
\hline $40-49$ & 0 & 502 & - & 0 & 188 & - \\
\hline $50-59$ & 6 & 749 & 0.8 & 0 & 323 & - \\
\hline $60-69$ & 28 & 1311 & $2 \cdot 1$ & 8 & 164 & 4.9 \\
\hline $70-74$ & 23 & 691 & $3 \cdot 3$ & 9 & 400 & $2 \cdot 2$ \\
\hline Total & 57 & 3253 & & 17 & 1075 & \\
\hline \multicolumn{7}{|c|}{ (b) Ostergötland county } \\
\hline $40-49$ & 2 & 566 & 0.4 & 0 & 343 & - \\
\hline $50-59$ & 8 & 959 & 0.8 & 5 & 617 & 0.8 \\
\hline $60-69$ & 14 & 1244 & $1 \cdot 1$ & 15 & 782 & 1.9 \\
\hline $70-74$ & 30 & 597 & 5.0 & 12 & 285 & $4 \cdot 2$ \\
\hline Total & 54 & 3366 & & 32 & 2027 & \\
\hline
\end{tabular}

1 Time at risk is calculated since operation 
the first 9 years of follow up derived from the first two screening rounds, since insufficient time has elapsed for the effect of later screening rounds to become apparent. The deficit of 58 breast cancer deaths in this age group has resulted from approximately 85000 mammographic examinations, one death prevented per 1460 mammographic examinations.

At the first screening round, some 14 biopsies were taken and 6.9 cancers detected per 1000 women screened among women aged 50-69. At the second screening round, the respective figures are 4.6 and $3 \cdot 5$. The deficit of 58 breast cancer deaths in this age group arose from nearly 800 biopsies and 443 breast cancers detected, a rate of one breast cancer death avoided per 13.5 biopsies, or per 7.4 breast cancer cases detected (including carcinoma in situ).

\section{Discussion}

The updated results strengthen the evidence on the reduction in breast cancer mortality following mass screening with mammography. Concern has been expressed about variation in its effect, between counties and age groups. The results above show that these variations are not statistically significant, that is, they are compatible with random variation. The test for a difference between the effects of screening in the two counties yields a $\chi^{2}$ statistic of 0.5 on one degree of freedom, so the observed variation is actually somewhat less than would be expected from pure chance. Further, the analysis of results from successive years shows the beneficial effect of screening in both counties remaining consistent and steadily gaining in significance. There is thus no evidence for a difference between the two counties in the effectiveness of screening.

Among the different age groups, some differences might be expected. Compliance in the group over 70 years of age at entry was much lower than in the younger age groups, so a decreased effect would be anticipated. In the group aged 40-49 years at entry, single view mammography appears to be less sensitive than in older age groups, again suggesting that the mortality effect may be lower. ${ }^{3} 11$ Nevertheless, no significant heterogeneity was seen $\left(\chi_{3}^{2}=2 \cdot 75\right.$, less than its expected value) and the results in tables 2 and 5 refer to the entire study population. Taken as a whole, screening is of benefit to the age group 40-74.

To answer unequivocally questions about specific subgroups, a new trial would have to be designed for each subgroup, although the results already appear convincing for the 50-69 age group. For the age group $40-49$, the new trial would have to be substantially larger than the present one to have acceptable power to detect the benefit as significant, due to the lower incidence and the small mortality rate from breast cancer in this age group. Adami et al ${ }^{12}$ found that women diagnosed as having breast cancer in this age group have two year relative survival rates of $90 \%$ and five year relative rates of $75 \%$. It might be argued from this that screening may not be cost effective in this group, even though it does confer a limited benefit in survival terms. The argument, however, does not begin and end with survival alone; early detection should also save many otherwise healthy women in their forties from disfiguring or debilitating therapy, in itself a worthwhile end.

Due to some imbalances in the age distribution in the ASP and the PSP, a small, non-significant excess of deaths from causes other that breast cancer in the ASP was observed. This excess disappeared after adjustment for county and age, so that mortality rates in the ASP and PSP were identical. This is reassuring, but should not occasion surprise in the absence of any plausible mechanism whereby such an excess could arise. The result does emphasise an important point about analysis of data such as these; when randomisation has taken place at the group level, small imbalances in baseline characteristics can arise in seemingly comparable groups and lead to minor biases. Age adjustment is invariably worthwhile.

One objection to the claims for the benefits of breast cancer screening is that no effect has been demonstrated on total mortality. ${ }^{4-9}$ This point merits some discussion. In the age group 40-74 years, breast cancer in Swedish women causes about $7 \%$ of deaths. A $30 \%$ reduction in breast cancer mortality would therefore lead to a $2 \%$ reduction on overall mortality, well within the confidence interval seen for the relative risk in table 10 after adjustment for age and county. Furthermore, after an average of 7.9 years follow up, the death rate from breast cancer diagnosed after the start of the trial (ie, the breast cancer deaths on which the $30 \%$ reduction is based) is still only about half the breast cancer mortality rate in the general population. The expected effect on total mortality seen so far is therefore only $1 \%$, exactly as seen in table 10 . This point underlines the naivety of those who ask for an observable and significant reduction in total deaths. In fact, very few new public health or therapeutic measures in western countries could be expected to show a significant reduction in total deaths; an outright ban on cigarette smoking might be the only example.

As regards the suggestion that different policies for classifying cause of death were exercised in the ASP and the PSP, ${ }^{9}$ we have demonstrated here that no evidence for this exists in the Swedish study. The rates for deaths from other causes among breast cases in the two groups are quite strikingly similar. Many factors other than breast cancer mortality contribute to a full evaluation of breast cancer screening. These include 
The benefit of being able to use less radical surgery on tumours detected earlier, the disadvantage of undue anxiety occasioned by publicity for screening programmes, and the cost, inconvenience and harm of negative biopsies. ${ }^{578}$ In a developed country, however, anxiety over breast cancer is already widespread, which the publicity for screening may well help to mitigate. With respect to negative biopsies, alarming figures are quoted from North America, ${ }^{8}$ with benign to malignant ratios at biopsy in the 6 to 10 range. In much of Europe, the situation is different. In this study, for example, in the first round of screening, $50 \%$ of biopsies were cancers, and in the second round $75 \%$ were cancers. ${ }^{1314}$ Similar figures are reported from Holland. ${ }^{15}$

As regards financial cost, it is estimated that the Swedish project costs $\$ 3400$ per year of life saved, reasonable in comparison with the costs of other public health measures. ${ }^{16}$

We thank Ulla Brith Krusemo of Uppsala University Computing Centre and Mark Ansell of the South Bank Polytechnic, London, for computing assistance. Iris Castleton typed the manuscript and tables.

This research was supported by a grant from Kopparberg County Council, Sweden. The additional members of the project group in Kopparberg were A Cohen, Department of Surgery, A Gad, Department of Pathology, and U Ljungquist, Department of Surgery, Central Hospital, Falun, Sweden. Additional members in Östergötland were L G Arnesson, Department of Surgery, L Baldetorp, Department of Oncology, O Gröntoft, Department of Pathology, and J C Mansson, Department of Clinical Cytology, University Hospital, Linköping, Sweden.

Address for correspondence and reprints: Dr Laszlo Tabar, Department of Mammography, Central Hospital, 79182 Falun, Sweden

\section{Appendix}

Guidelines for classification of death as being from breast cancer, Swedish two county trial

To be considered as potential breast cancer deaths, breast cancer must be confirmed by histology or cytology. If this requirement is specified, and the subject is not lost to follow up (see table A1), the following four possibilities arise:

1. DISTANT METASTASES DEMONSTRATED PRIOR TO DEATH-AUTOPSY NOT PERFORMED.

The methods considered valid for demonstration of distant metastases prior to death are listed in table A2. If there was no evidence of another malignant disease, breast cancer was included as a primary or contributing cause of death (table $\mathrm{A} 1$, number 2 ). If there was evidence of another malignant disease and the clinical picture could not unequivocally determine the origin of metastases, breast cancer was considered to be a cause of death. If there was evidence of another malignant disease, with strong clinical evidence that the metastases originated from the other malignant disease, breast cancer was not considered to be a cause of death (table $\mathrm{Al}$, number 4).

2. DISTANT METASTASES DEMONSTRATED PRIOR TO DEATH-AUTOPSY PERFORMED.

If there was no evidence of other malignant disease, breast cancer was included as one of the causes of death, primary or contributing (table A1, number 2). If there was evidence of another malignant disease and the autopsy findings could not unequivocally determine the origin of metastases, breast cancer was considered as one of the causes of death (table Al, number 2). If there was evidence of another malignant disease and the autopsy findings unequivocally determined the origin of metastases to be the other malignant disease, breast cancer was not considered a cause of death (table Al, number 4).

3. NO EVIDENCE OF DISTANT METASTASES PRIOR TO DEATH-AUTOPSY NOT PERFORMED.

If clinical examination showed local or regional recurrent (or residual) breast cancer, the patient was considered to have died of intercurrent disease with breast cancer present (table A1, number 3A). Such cases occurred very rarely. Otherwise breast cancer was not considered to be a cause of death (table Al, number 4).

4. NO EVIDENCE OF DISTANT METASTASES PRIOR TO DEATH-AUTOPSY PERFORMED.

If there was no evidence of malignant disease at autopsy, breast cancer was not considered to be a cause of death (table A1, number 4). If there was evidence of another malignant disease and autopsy did not unequivocally determine the origin of metastases, breast cancer was considered to be a cause of death (table A1, number 2). If the autopsy determined the origin unequivocally as from the other malignant disease, breast cancer was not considered to be a cause of death (table Al, number 4). If the autopsy demonstrated only local or regional recurrent or residual breast cancer, the patient was considered to have died of intercurrent disease with breast cancer present (table A1, number 3A). Again, this was very rare. If the autopsy demonstrated only a solitary, localised distant metastasis, which was not symptomatic ante mortem and which could not have led to death, the patient was considered to have died of 
intercurrent disease with breast cancer present (table Al, number 3B).

In published analyses, including the present one, groups 2, 3A and 3B are all classed as breast cancer deaths. The inclusion of $3 \mathrm{~A}$ and $3 \mathrm{~B}$ may have exaggerated the numbers of breast cancer deaths in the screened group, indicating that our improvement in breast cancer mortality from screening is a conservative estimate. We plan to perform a more detailed analysis of the individual causes in table $A 1$ in the future.

Table Al Causes of death

1. Lost to follow up

2. Breast cancer death; breast cancer is the primary or the contributory cause of death

3. Death from intercurrent disease; limited residual/recurrent breast cancer present, clinically not a contributory cause of death:

(a) local and/or regional, recurrent and/or residual breast cancer

(b) solitary, asymptomatic, localised distant metastasis

4. Death from intercurrent disease; no data on presence of breast cancer at the time of death

Table A2 Methods of demonstration of distant metastases prior to death

Clinical examination methods

1. Chest $x$-ray

2. Radionuclide bone scan with verification by $x$-ray

3. Bone x-ray

4. Computed tomography (liver, brain)

5. Ultrasound (liver)

Liver: CT and/or ultrasound and radionuclide liver scan

Brain: CT

Histological or cytological examination

eg, from pleural effusion, from ascites, skin metastasis, liver biopsy, etc.

\section{References}

1 Tabar L, Fagerberg CJG, Gad A, et al. Reduction in mortality from breast cancer after mass screening with mammography. Lancet 1985; i: 829-32.

2 Tabar L, Gad A. Screening for breast cancer: the Swedish trial. Radiology 1981; 138: 219-22.

${ }^{3}$ Fagerberg G, Baldetorp L, Gröntoft O, Lundström B, Månson $\mathrm{JC}$, Nordenskjöld $\mathrm{B}$. Effects of repeated mammographic screening on breast cancer stage distribution. Acta Radiol Oncol 1985; 24: 465-73.

${ }^{4}$ Skrabanek P. Breast cancer screening. Lancet 1985; ii: 94.

${ }^{5}$ Skrabanek P. False premises and false promises of breast cancer screening. Lancet 1985; ii: 316-20.

${ }_{7}^{6}$ Skrabanek P. Breast cancer screening. Lancet 1985; ii: 941.

${ }^{7}$ Wright CJ. Should the hunt for non-palpable breast lesions be called off? Can J Surg 1986; 29: 299-300.

${ }^{8}$ Wright CJ. Breast cancer screening: a different look at the evidence. Surgery 1986; 100: 594-8.

9 Isacsson SO, Larsson LG, Janzon L. Är dokumentationen verkligen tillräcklig? Forcera inte fram screening utan debatt. Läkartidningen 1985; 82: 2672-3.

${ }^{10}$ Berry G. The analysis of mortality by the subject-years method. Biometrics 1983; 39: 173-84.

11 Tabar L, Fagerberg G, Day NE, Holmberg L. What is the optimum interval between mammographic screening examinations?-An analysis based on the latest results of the Swedish two-county breast cancer screening trial. Brit $J$ Cancer 1987; 55: 547-51.

12 Adami H-O, Malker B, Holmberg L, Persson I, Stone B. The relation between survival and age at diagnosis in breast cancer. $N$ Engl J Med 1986; 315: 559-66.

${ }^{13}$ Andersson I, Fagerberg G, Lundgren B, Tabar L. Breast cancer screening in Sweden: the single modality approach. Radiologe 1980; 20: 608-11.

${ }^{14}$ Swedish National Board of Health and Welfare. Mammografiscreening: Hälsokontroll för tidig upptäckt av bröstcancer. Stockholm: Socialstyrelsen, 1986.

15 Day NE, Chamberlain J. Screening for breast cancer: workshop report. Eur J Cancer Clin Oncol 1988; 24: 55-9.

${ }^{16}$ Day NE, Baines CJ, Chamberlain J, Hakama M, Miller AB, Prorok P. UICC project on screening for cancer: report of the workshop on screening for breast cancer. Int J Cancer 1986; 38: 303-8.

Accepted for publication February 1989 